\title{
MOTION OF A POINT VORTEX IN A MULTICONNECTED REGION*
}

BY

AMBADY SURESH

Princeton University

Abstract. The motion of a single point vortex in a bounded region of the plane with $m$ internal boundaries is considered. For $m \geqslant 1$, it is shown that there exist $m-1$ saddle points (counted with multiplicities) where the vortex can remain stationary.

In this paper we consider the motion of a cylindrical point vortex confined to a bounded region $A$ of the plane $R^{2}$. This region is internally bounded by fixed Jordan curves $C_{k}(k=1,2, \ldots, m)$ and bounded externally by a Jordan curve $C_{0}$. The interior of $A$ is filled with inviscid incompressible fluid undergoing irrotational motion.

If $(x, y)$ are the coordinates of the vortex and its strength unity, then Lin [1] has shown that a Hamiltonian $H(x, y)$ exists such that the motion of the vortex is given by

$$
\frac{d x}{d t}=-\frac{\partial H}{\partial y}, \quad \frac{d y}{d t}=\frac{\partial H}{\partial x}
$$

where

$$
H(x, y)=\frac{1}{2} g(x, y ; x, y)=\frac{1}{2} \operatorname{Lim}_{\substack{x_{0} \rightarrow x \\ y_{0} \rightarrow y}} g\left(x, y ; x_{0}, y_{0}\right) .
$$

The function

$$
g\left(x, y ; x_{0}, y_{0}\right)=G\left(x, y ; x_{0}, y_{0}\right)-\frac{1}{2 \pi} \log \left[\left(x-x_{0}\right)^{2}+\left(y-y_{0}\right)^{2}\right]^{1 / 2}
$$

is harmonic with respect to $(x, y)$ throughout the region $A$ including the point $\left(x_{0}, y_{0}\right)$ and $G\left(x, y ; x_{0}, y_{0}\right)$ satisfies the following boundary conditions in $A$ :

1) $G=A_{k}=$ constants on $C_{k}$

$$
\oint_{C_{k}} \frac{\partial G}{\partial n} d s=0
$$

2) $G=0$ on $C_{0}$.

The vortex trajectories are given by $g(x, y ; x, y)=$ const. These trajectories may intersect at a "saddle point" where the vortex can remain stationary. At such a point

${ }^{*}$ Received June 23, 1983. 
$\left(x_{0}, y_{0}\right)$ we have

$$
\frac{\partial H}{\partial x}=\frac{\partial H}{\partial y}=0 ; \quad H\left(x_{0}, y_{0}\right)=C .
$$

The saddle point is of multiplicity $p$ if in a small neighborhood of $\left(x_{0}, y_{0}\right)$,

$$
H(x, y)-C=O\left(r^{p+1}\right)
$$

where $r$ is the radius vector from $\left(x_{0}, y_{0}\right)$. We will show that:

THEOREM. If $m$ is the number of internal boundaries then for $m \geqslant 1$, there are precisely $m-1$ saddle points (counted with multiplicities) inside $A$.

Proof.

Assertion 1. A vortex at the interior of $A$ cannot touch the boundary.

To show this, simply note that $H(x, y)$ is regular everywhere inside $A$, whereas on $\partial A$ it is logarithmically infinite (cf. equation (2)). Since $H$ is an integral of the motion, this establishes Assertion 1.

Assertion 2. There exists a harmonic function $\psi(x, y)$, regular in $A$, and constant on $\partial A$, whose level curves are the trajectories of the vortex. This step is necessary because the function $g(x, y ; x, y)$ is not necessarily harmonic. Consider, for example, the exterior problem for the unit circle. The function $g\left(x, y ; x_{0}, y_{0}\right)$ satisfying conditions above is easily derived as

$$
g(r, \theta ; \rho, \phi)=-\frac{1}{2 \pi} \ln \left[1+\frac{1}{\rho^{2} r^{2}}-\frac{2}{\rho r} \cos (\theta-\phi)\right]^{1 / 2}
$$

and consequently the function $g(x, y ; x, y)$ is

$$
g(r, \theta ; r, \theta)=-\frac{1}{2 \pi} \ln \left[1-\frac{1}{r^{2}}\right]
$$

which is not harmonic. Nevertheless, the trajectories of the vortex are circles, and are the level curves of the harmonic function $\psi=\ln r$, which is regular inside $A$ and constant on $\partial A$.

To see that the assertion is true in general, note that equations (1) imply that the vortex at $P\left(x_{0}, y_{0}\right)$ moves with the fluid velocity induced at $P$ due to external agencies. Thus its motion is identical to that of a fluid particle situated at $P$. Hence, vortex trajectories correspond to some fluid motion in the given region whose stream function we will call $\psi(x, y) . \psi(x, y)$ is some superposition of the image system whose streamfunction is $g\left(x, y ; x_{0}, y_{0}\right)$ which is regular inside the given region. Hence $\psi(x, y)$ is regular in $A$. The assertion remains true when $A$ is simply connected $(m=0)$ but in this case, $\psi(x, y)$ would be trivially constant all over $A$.

The theorem now follows from well-known results due to Morse [2] on the topology of harmonic functions, applied to the function $\psi(x, y)$. In particular, from Theorem 13.2 of [2], if

$$
\begin{aligned}
& S=\text { no. of saddle points (counted with multiplicities) } \\
& p=\text { total number of bounding curves }=m+1
\end{aligned}
$$




$$
\begin{aligned}
\sigma_{i}= & \text { level indices of each boundary (this is briefly, } \\
& \text { half the number of curves that touch } C_{\mathrm{i}} \text { ). }
\end{aligned}
$$

then

$$
S=p-2-\sum_{i=1}^{m} \sigma_{i} .
$$

By Assertion 1, the level index of each boundary is zero and the theorem follows.

Let us briefly indicate some consequences of the above theorem. When the critical points are all of multiplicity one (i.e. nondegenerate), then each critical point is an unstable saddle. For large values of $H(x, y)$, the trajectories are closed curves around each internal and external boundary. As a critical value of $H$ is crossed, two of these closed curves merge via the saddle point. In this way, the topology of the trajectories can be determined by the location and level of each critical point.

More importantly, a complete classification of trajectories is possible. Let $p$ be an interior point of $A$. Then the trajectory through $p$ is one of three types as given below:

(1) $\nabla H=0$ at $p$. The trajectory is the single point $p$.

(2) $\nabla H \neq 0$ at $p, H(p)=H^{*}$ a critical value. The trajectory begins and ends at a critical point.

(3) $\nabla H \neq 0$ at $p$ and $H(p) \neq H^{*}$. The trajectory is a closed curve.

Statements (1) and (2) are obvious and statement (3) follows from the fact that the trajectory is the inverse image of a regular value, and hence is a compact one-dimensional manifold without boundary (cf. Assertion 1). Hence it must be diffeomorphic to the circle.

In conclusion, we mention a few related open problems. Generically, all saddle points will be nondegenerate (i.e. of multiplicity one), since such functions are open and dense in the class of functions defined on $A$. Since every nondegenerate critical point is unstable, a stable equilibrium point, if it exists, must be degenerate and hence exceptional. Whether such stable equilibrium points exist at all is still an open question to the best of the knowledge of this author. Lastly, no such results on equilibrium points are known for the interesting case where there are fixed boundaries extending to infinity for which the Hamiltonian formulation is still valid.

Note Added in Proof: In addition to the $n-1$ saddle points, there always exists at least one closed curve consisting entirely of equilibrium points. To see this, note that assertion 2 implies that $H=H(\psi)$. From the boundary conditions on $H$ and $\psi$, it is clear that $H^{\prime}(\psi)=0$ for some intermediate $\psi_{0}$. Then, the curve $\psi(x, y)=\psi_{0}$ consists entirely of equilibrium points.

\section{REFERENCES}

[1] C. C. Lin, Proc. Natl. Acad. Sci., USA 27, 570-75 (1941)

[2] M. Morse, Topological Methods in the Theory of Functions of a Complex Variable, Ann. of Math. Studies 15, Princeton Univ. Press 